\title{
Estilos parentais e uinculação amorosa: efeito mediador do bem-estar psicológico em jovens adultos
}

\author{
Parenting Styles and Romantic fittachment: The Mediating Effect \\ of Psychological Well-being in Young Adults \\ Estilos parentales y uinculación amorosa: efecto mediador \\ del bienestar psicológico en jóvenes adultos
}

\author{
Maria Bárbara Granja*, Catarina Pinheiro Mota \\ Universidade de Trás-os-Montes e Alto Douro
}

Doi: http://dx.doi.org/10.12804/revistas.urosario.edu.co/apl/a.5584

\section{Resumo}

A vinculação às figuras primordiais e os estilos parentais assumem especial relevância na forma como os jovens adultos estabelecem as suas relações e transições ao longo do seu percurso afetivo. As relações satisfatórias entre a díade pais-filhos exercem um papel preponderante no estabelecimento de relações amorosas seguras e na manutenção ou aumento do bem-estar psicológico do jovem adulto. O presente estudo tem como principal objetivo analisar o efeito dos estilos parentais na vinculação amorosa de jovens adultos, assim como testar o papel mediador do bem-estar psicológico na associação anterior. A amostra foi constituída por 787 jovens universitários com idades compreendidas entre os 18 e os 25 anos. A recolha de dados foi realizada com recurso a instrumentos de autorrelato, nomeadamente, Parental Styles and Dimensions Questionnaire: Short Version (PSDQ), Questionário de Vinculação Amorosa e a Escala de Medida de Manifestação do Bem-estar Psicológico. Os resultados apontam para um efeito preditor positivo do estilo parental democrático relativamente ao bem-estar psicológico e à confiança na vinculação amorosa, assim como um efeito negativo face à ambivalência e dependência da vinculação amorosa. Verifica-se ainda, o papel do bem-estar psicológico enquanto variável mediadora na associação entre um estilo parental democrático e a vinculação amorosa. Palavras-chave: vinculação, estilos parentais, vinculação amorosa, bem-estar psicológico.

* Mestre em Psicologia Clínica. Universidade de Trás-os-Montes e Alto Douro - UTAD, Edifício da Escola de Ciências Humanas e Sociais - Polo I. Quinta dos Prados - UTAD, 5000-801 Vila Real, Portugal. e-mail: barbaragranja@hotmail.com

Professora Auxiliar. Departamento de Educação e Psicologia, Universidade de Trás-os-Montes e Alto Douro - UTAD, Edifício da Escola de Ciências Humanas e Sociais - Polo I. Quinta dos Prados - UTAD, 5000-801 Vila Real, Portugal. Membro Integrado do Centro de Investigação de Psicologia da Universidade do Porto. e-mail: catppmota@utad.pt

Financiamento: This research was partially funded by FCT under the project

PEst-C/PSI/UI0050/2011 and FEDER funds through the COMPETE program under the project FCOMP-01-0124-FEDER-02271

Cómo citar este artículo: Granja, M. B., \& Mota, C. P. (2018). Estilos parentais e vinculação amorosa: efeito mediador do bem-estar psicológico em jovens adultos. Avances en Psicología Latinoamericana, 36(1), 93-109. doi: http://dx.doi.org/10.12804/ revistas.urosario.edu.co/apl/a.5584 


\section{fbstract}

Primordial figures and parenting styles are especially relevant to the way that young adults establish relationships and transitions over their emotional journeys. Satisfactory relations within the parent-child dyad play a leading role in establishing secure romantic relationships and maintaining or increasing the psychological well-being of young adults. This study aims to analyze the effect of parenting styles on the romantic relationships of young adults and to test the mediating role of psychological well-being in the parent-child association. The sample included 787 university students between 18 and 25 years of age. Data collection was performed using the following self-reporting instruments: the Parental Styles and Dimensions Questionnaire: Short Version (PSDQ), the Romantic Attachment Questionnaire, and the Psychological Well-being Measurement Scale. The results point to a positive predictor effect of democratic parenting styles in relation to psychological well-being and trust in romantic attachments, as well as a negative effect on ambivalence regarding romantic attachment and dependence on that attachment. Psychological well-being also functions as a variable mediating the association between democratic parenting styles and romantic attachment.

Keywords: Attachment, parenting styles, romantic attachment, psychological well-being.

\section{Resumen}

La vinculación a las figuras primordiales y los estilos parentales asumen especial relevancia en la forma como los jóvenes adultos establecen sus relaciones y transiciones a lo largo de su recorrido afectivo. Las relaciones satisfactorias entre el par padre-hijos ejercen un papel preponderante en el establecimiento de las relaciones amorosas seguras y en el mantenimiento o aumento del bienestar psicológico del joven adulto. El presente estudio tiene como principal objetivo analizar el efecto de los estilos parentales en la vinculación amorosa de jóvenes adultos, así como de probar el papel mediador del bienestar psicológico en la asociación anterior. La muestra fue constituida por 87 jóvenes universitarios con edades comprendidas entre los 18 y los 25 años. La recolección de datos fue realizada recurriendo a instrumentos de autorelato, principalmente Parental Styles and Dimensions Questionnaire: Short Version (PSDQ), Cuestionario de Vinculación Amorosa y la Escala de Medida de Manifestación del Bienestar Psicológico. Los resultados apuntan para un efecto predictor positivo del estilo parental democrático relativamente al bienestar psicológico y a la confianza en la vinculación amorosa, así como un efecto negativo frente a la ambivalencia y dependencia de la vinculación amorosa. Se comprueba, además, el papel del bienestar psicológico como variable mediadora en la asociación en un estilo parental democrático y la vinculación amorosa.

Palabras clave: vinculación, estilos parentales, vinculación amorosa, bienestar psicológico.

Considerando que a relação com as figuras parentais exerce um papel no desenvolvimento dos jovens adultos, a teoria da vinculação parece assumir relevância na relação entre os estilos parentais e a forma como os jovens gerem as suas relações e transições ao longo do seu percurso afetivo. A vinculação é caraterizada como um laço afetivo significativo que é estabelecido entre a criança e a figura cuidadora primordial, constituindo ao longo da infância e juventude uma fonte de proteção, conforto e apoio (Bolwby, 1988). Neste sentido, Bowlby (1969) defende que os vínculos seguros e inseguros estabelecidos entre a díade pais-filhos poderão ter um efeito na qualidade das relações interpessoais estabelecidas pela criança ao longo do seu desenvolvimento. Ao longo do desenvolvimento da adolescência e jovem adultícia os modelos internos dinâmicos vão sofrendo reorganizações à medida que se estabelecem novas relações (Bartholomew \& Shaver, 1998). Assim, partindo dos pressupostos da teoria da vinculação, Maccoby e Martin (1983) propuseram um modelo dos estilos parentais que evidenciou duas dimensões: a exigência e a responsividade. A primeira 
inclui todas as atitudes das figuras parentais que procuram controlar o comportamento dos filhos, tentando estabelecer limites e regras. A responsividade refere-se às atitudes compreensivas que as figuras parentais demonstram para com os seus filhos, expressando apoio emocional e desenvolvimento de autonomia e da auto-afirmação dos jovens. Desta forma, as figuras parentais com elevada responsividade e exigência são designados como democráticos, aqueles que apresentam baixa responsividade e exigência são tidos como figuras parentais negligentes. Acrescem a esta classificação as figuras parentais muito responsivas, mas pouco exigentes que são definidas como indulgentes; por outro lado, aquelas figuras parentais que são muito exigentes e pouco responsivas são categorizadas como autoritárias (Maccoby \& Martin, 1983).

Baumrind (1991) postula que os estilos parentais poderão exercer um contributo significativo no que concerne ao percurso desenvolvimental do indivíduo e nas relações afetivas que são estabelecidas com os outros significativos e consigo mesmo. O estilo parental permissivo é geralmente caraterizado como fazendo parte dele pais que evitam exercer um controle sobre os filhos, que realizam poucas exigências, e no qual a obediência não é valorizada. Geralmente, proporcionam autonomia aos filhos, para que tomem suas próprias decisões, bem como explicam as regras a serem cumpridas no ambiente familiar (Baumrind, 1966, 1968). Os pais que exercem um estilo parental autoritário, geralmente avaliam e controlam os comportamentos dos filhos de acordo com padrões de conduta rígidos e inflexíveis. A comunicação na díade pais-filhos é caraterizada por uma baixa responsividade e rejeição, não havendo desta forma a valorização do diálogo devido ao elevado grau de exigência e controle parental (Baumrind, 1966, 1968). Já os pais que adotam um estilo parental democrático, tendem a estabelecer limites e regras de forma racional e orientada, valorizando um controle firme, gerando padrões familiares saudáveis e equilibrados. A comunicação pais-filhos é baseada no respeito mútuo e os pais valorizam muito a tomada de decisões por parte dos filhos. Desta forma, a exigência, a autonomia, o estímulo à comunicação, o uso do poder de forma equilibrada e não punitiva, a responsividade são caraterísticas enraizadas neste estilo parental (Baumrind, 1966, 1968; Baumrind, Larzelere \& Owens, 2010; Cecconello, Antoni \& Koller, 2003).

Os estilos parentais sugerem ser relevantes no estabelecimento de relações de vinculação com o par romântico. O desenvolvimento saudável de relações românticas na vida adulta poderá ser predito por uma vinculação segura e pela percepção de satisfação e confiança, que recria nos jovens disponibilidade para o par amoroso (Altin \& Terzi, 2010; Fraley, 2002).Todavia existem fatores que parecem contribuir para esta vinculação ao par romântico, nomeadamente as experiências de segurança ou insegurança que são vivenciadas à medida que a relação com o par romântico evolui (Dinero, Conger, Shaver, Widaman \& Larsen-Rife, 2008; Shaver \& Hazan, 1988). Numa fase inicial, o par amoroso desempenha a função de porto seguro, este é caraterizado como uma figura afetivamente significativa à qual se recorre em situação de necessidade, procurando conforto, proteção, apoio e alívio (Ainsworth, 1991). Na idade adulta, em geral, a relação com o par amoroso pode constituir-se numa relação de vinculação, proporcionando sentimentos de segurança, na medida em que promove a exploração do mundo e gera um sentimento de competência pessoal (Shaver \& Hazan, 1988). Com base nesta premissa, o indivíduo poderá sentir-se mais seguro ao percecionar o seu parceiro como acessível e responsivo, sendo que este poderá vir a desempenhar um papel relevante enquanto fonte de segurança e proteção em momentos ameaçadores (Hazan \& Shaver, 1990). Desta forma, os estilos parentais parecem criar expectativas face à forma como os jovens se dispõem nas relações que estabelecem com os outros e nas estratégias de regulação emocional que estabelecem face às relações de proximidade 
(Gleeson \& Fitzgerald, 2014; Matos \& Costa, 2006; Muraru \& Turluc, 2012).

\section{Papel do bem-estar psicológico na associação entre estilos parentais e vinculação amorosa em jovens adultos}

Tal como abordado anteriormente, os estilos parentais parecem ser relevantes na forma como adolescentes e jovens adultos gerem suas vivências afetivas, assumindo esta questão particular relevância, quando abordada a dinâmica das relações amorosas. Em termos ilustrativos, o estilo parental permissivo parece ser pautado por ausência de regras, baixa exigência, ajuda excessiva e pouca estimulação da criança. Esta vivência poderá ocasionar falta de autocontrole e de autoconfiança, que podem levar os filhos ao sentimento de dependencia e superproteção (Baumrind, 1966, 1968). O controle parece limitar o desenvolvimento da autonomia, uma vez que as necessidades do indivíduo são suprimidas. Como consequência desta prática parental, ocorrem comportamentos dependentes, reações passivo-agressivas e baixas motivações para realizações (Baumrind, 1980). Esta questão é particularmente relevante nas relações com pares românticos. Nesta fase, ocorre o processo de separação-individuação decorrente dos laços afetivos estabelecidos com as figuras parentais e na relação amorosa. O processo de separação-individuação face às figuras parentais assume relevância, dando destaque à novas relações fora do seio familiar, nomeadamente na relação amorosa. Na jovem adultícia destaca-se a procura de confiança, suporte, apoio emocional constituindo desta forma domínios da individuação, iniciando-se as relações de vinculação na relação romântica (Komidar, Zupančič, Sočan \& Puklek Levpušček, 2014). Neste sentido, também as relações românticas parecem ser relevantes ao longo do processo de separação-individuação (e.g. Mattanah, Hancock \& Brand, 2004; Mota \& Rocha, 2012; Saraiva \& Matos, 2012).
As relações românticas desempenham um papel fundamental no desenvolvimento do jovem adulto, na medida em que satisfazem necessidades afetivas (Collins, Welsh \& Furman, 2009). Quando confrontado com eventos ansiogênicos, o jovem adulto procura proteção e apoio recorrendo ao seu sistema de vinculação, nomeadamente as figuras primordiais e o par romântico. As figuras significativas que transmitam segurança ao jovem adulto parecem gerar um efeito positivo no bem-estar psicológico do mesmo (Buist, Dekovic, Meeus \& VanAken, 2002; Veld \& Denollet, 2011). Segundo Massé et al. (1998) o bem-estar psicológico refere-se à presença de sinais de felicidade, autocontrole, autoestima, equilíbrio, sociabilidade e envolvimento social, e não apenas à ausência de sintomas tais como ansiedade ou depressão manifestados pelo indivíduo. Deste modo, o bem-estar psicológico pode estar relacionado com o envolvimento positivo que o indivíduo estabelece com os outros (Massé et al.,1998).

De acordo com Pinto (2009), o bem-estar psicológico pode ser promovido pelas relações românticas dos jovens adultos, sendo que estas podem proporcionar aprendizagens e o desenvolvimento de estratégias de forma a lidar com sentimentos de aceitação, com as adversidades, autoestima e felicidade. Williams e Riskind (2004) elaboraram um estudo com 291 sujeitos, que teve como objetivo principal examinar o tipo de vinculação amorosa nas relações entre adultos, vulnerabilidades cognitivas relativas à ansiedade e depressão e sintomas ansiosos e depressivos. Os resultados demonstraram que uma vinculação insegura ao par amoroso estava relacionada com níveis mais elevados de vulnerabilidade cognitiva, a níveis mais elevados de sintomas psicopatológicos e a mais problemas ao nível do relacionamento. Neste sentido, existem diferenças entre indivíduos dependendo do tipo de estilo parental a que foram sujeitos, bem como ao nível do bem-estar psicológico e da vinculação amorosa que estabelecem com o par romântico. 


\section{Objetivos e hipóteses}

O presente estudo tem como principal objetivo analisar o efeito dos estilos parentais na vinculação amorosa e, testarem em que medida o bem-estar psicológico pode exercer um efeito mediador na associação anterior. Pretende-se ainda analisar as diferenças dos estilos parentais e da vinculação amorosa em função das dimensões sociodemográficas da amostra, nomeadamente idade e o sexo.

Neste sentido, espera-se que existam diferenças estatisticamente significativas da vinculação amorosa e dos estilos parentais face ao sexo e idade. Prevê-se que um estilo parental democrático prediga positivamente o bem-estar psicológico. E por último, espera-se que ocorra um efeito mediador do bem-estar psicológico na associação entre um estilo parental democrático e a vinculação amorosa.

\section{Método}

\section{Participantes}

No estudo participaram 787 jovens universitários, pertencentes à Universidade de Trás-os-Montes e Alto Douro (Vila Real, Portugal), e pertencentes ao Instituto Politécnico de Viana do Castelo (Portugal), com idades compreendidas entre os 18 e os 25 anos ( $M=20,07, D P=1,708 \%)$, dos quais $233(29,6 \%)$ são do sexo masculino e $554(70,04 \%)$ do sexo feminino. Relativamente à relação amorosa, 395 sujeitos (50,2\%) reportam ter um relacionamento amoroso cuja duração varia entre 1 mês e 108 meses $(\mathrm{M}=12,15, \mathrm{DP}=20,22)$, 392 sujeitos $(40,8 \%)$ referem que já namoraram, mas neste momento não namoram, sendo que a duração média da relação anterior foi de 21 meses $(\mathrm{M}=21,04, \mathrm{DP}=21,46)$. Desta forma, a amostra é constituída por indivíduos cujas relações amorosas foram consideradas estáveis.

\section{Instrumentos}

Questionário Sociodemográfico - foi construído um questionário sociodemográfico que permitiu obter informações relativas à idade, sexo e recolher dados acerca do meio familiar e relação amorosa.

O Parental Styles and Dimensions Questionnaire: Short Version $(P S D Q)$ foi traduzido para a população portuguesa por Nunes e Mota (in press), a partir da versão original de Robinson, Mandleco, Olsen \& Hart (1995). Consiste numa versão reduzida composta por 32 itens, o instrumento original era composto por 133 itens. Este questionário apresenta uma versão para a "Mãe" e outra para o "Pai" em que apenas difere o gênero na formulação das afirmações. É um instrumento de heterorrelato (respondido pelos jovens face à percepção dos estilos parentais) no qual as respostas se apresentam numa escala tipo Likert que varia entre 1 - "Nunca" e 5 - "Sempre". As respostas são elaboradas tendo em conta a frequência com que ocorrem as situações referidas nos itens. $\mathrm{O}$ instrumento organiza-se em três dimensões: o estilo democrático, o autoritário e o permissivo, que vão de encontro à tipologia de Baumrind (1971). Cada dimensão organiza-se em subdimensões, sendo que, do estilo democrático (Dem.) fazem parte as escalas: apoio e afeto, regulação e cedência de autonomia; do estilo autoritário (Aut.) fazem parte as escalas: coerção física, hostilidade verbal e punição; e do estilo permissivo (Per.) apenas a escala indulgência. Ainda que esta seja a organização original do instrumento, neste estudo, tendo em conta as propriedades psicométricas, procedeu-se à agregação das dimensões coerção física e punição numa só, uma vez que sob o ponto de vista semântico e estrutural obtiveram-se índices de ajustamento mais adequados à amostra em estudo. Destaca-se ainda que, através das análises confirmatórias de $1^{\mathrm{a}}$ ordem, se constatou que a dimensão hostilidade verbal, nesta amostra, não se encontra ajustada face ao constructo originalmente estabelecido pelo autor, sendo que foi retirada da análise para não 
enviesar os resultados. Analisados os valores relativos às dimensões, verificam-se valores de alfa de 0,95 e 0,93 no estilo democrático, 0,77 e 0,77 no estilo autoritário e 0,62 e 0,60 no estilo permissivo, quanto ao Pai e à Mãe, respectivamente. Quanto às análises fatoriais confirmatórias verificaram-se índices de ajustamento adequados para os modelos $x^{2}(78)=334,55, p=0,001, x^{2}(77)=360,14, p$ $=0,001$, Ratio $=4,29 / 5.42$, SRMR $=0,093 / 0,081$, RMSEA $=0,07 / 0,07, \mathrm{CFI}=0,97 / 0,95$ quer da versão da mãe, quer do pai, respectivamente, os modelos apresentaram índices de ajustamento dentro dos valores de referência.

Escala de Medida de Manifestação do Bem-estar Psicológico (traduzida e adaptada para a população portuguesa por Monteiro, Tavares \& Pereira, 2007, a partir da versão original da Échelle de Mesure dês Manifestations du Bien- Être Psychologique de Massé, Poulin, Dassa, Lambert, Bélair \& Battaglini, 1998). Esta escala é constituída por 25 itens, organizados em 6 escalas que avaliam fatores como autoestima, equilíbrio, envolvimento social, sociabilidade, controle de si e acontecimentos, felicidade. A resposta aos itens é atribuída numa escala tipo Likert, que varia de 1 - "Nunca" a 5 - "Quase Sempre". Relativamente à análise por dimensão verificam-se valores de .91 na dimensão felicidade, .85 na dimensão autoestima, .87 na dimensão controle de si e dos acontecimentos, .71 na dimensão envolvimento social, .75 na dimensão equilíbrio e .85 na dimensão sociabilidade. Quanto às análises fatoriais confirmatórias, verificaram-se índices de ajustamento adequados para os modelos $\mathrm{x}^{2}(120)=487,30, \mathrm{p}=0,001$, Ratio $=4,06, \mathrm{RMR}=$ 0,03, RMSEA $=0,06$, CFI $=0,96$.

Questionário de Vinculação Amorosa (QVA) validado para a população portuguesa por Matos, Barbosa e Costa (2001). Tem como objetivo avaliar a percepção dos adolescentes e jovens adultos relativamente à qualidade do vínculo com o seu parceiro amoroso. Neste estudo utilizou-se a versão reduzida do instrumento, constituída por 25 itens (Matos, Barbosa \& Costa, 2001), que se organizam em quatro dimensões relativas ao par amoroso, enquanto figura de vinculação. Assim sendo, as dimensões do questionário são: a confiança, a dependência, o evitamento e a ambivalência. Para responder ao questionário o sujeito deve focar-se na sua relação atual, caso exista, ou naquela que no passado foi a mais duradoura. Caso o sujeito ainda não tenha tido uma relação, que considere de namoro, lhe é pedido que imagine como seria a sua relação com um namorado(a) ou então que se reporte a relações em que tenha "curtido". As suas respostas são dadas numa escala tipo Likert, que varia entre 1 - "Discordo totalmente" e 6 "Concordo totalmente". Relativamente à análise por dimensão, verificam-se valores de 0,82 na confiança, 0,75 na dependência, 0,77 no evitamento e 0,83 na ambivalência. Quanto às análises fatoriais confirmatórias verificaram-se índices de ajustamento adequados para os modelos $x^{2}(48)$ $=233,11, p=0,001$, Ratio $=4,86$; RMR $=0,05$; RMSEA $=0,07$; CFI $=0,95$, ou seja, os modelos apresentaram índices de ajustamento dentro dos valores de referência.

\section{Procedimento}

A recolha de dados foi realizada de forma aleatória em diversos cursos, anos e turmas de instituições de ensino superior da zona norte de Portugal que aceitaram colaborar na investigação. Na fase anterior procedeu-se ainda à validação semântica com 12 jovens entre os 18 e os 25 anos. A recolha de dados decorreu entre Outubro de 2015 e Janeiro de 2016, em contexto de sala de aula, na presença do investigador responsável. O protocolo (parecer da comissão de ética N 22/2015, doc.17/CE/2017, atribuído pela comissão de ética da Universidade de Trás-os-Montes e Alto Douro) foi submetido ao conselho de ética da Universidade de Trás-Os-Montes e Alto Douro, como forma a garantir todos os pressupostos éticos inerentes ao projeto de investigação, obtendo aprovação. 


\section{Análises dos dados}

A avaliação dos sujeitos da amostra foi realizada num único momento, tratando-se, portanto, de uma investigação de caráter transversal, não estando prevista uma reavaliação ou seguimento dos sujeitos. O tratamento de dados foi realizado com recurso ao programa estatístico SPSS - Statistical Package for Social Sciences - na sua versão 22. Com esta análise confirmou-se que a amostra em estudo cumpria os critérios de normalidade e, portanto, definiu-se a realização de análises estatísticas mediante testes paramétricos (Maroco, 2007). Foi realizado um conjunto de análises estatísticas nas quais foram considerados valores de significância de $p<.05$ para a interpretação dos dados. Destaca-se o recurso a análises correlacionais e análises de variância multivariadas (MANOVAS). Os efeitos preditores foram testados com recurso a análises de regressão múltipla hierárquica e com modelos de equações estruturais, combinando variáveis latentes com path análises (Maroco, 2014). O teste de mediação foi realizado com o programa AMOS, com recurso ao método de bootstraping. Este método tem por finalidade realizar inferências para os efeitos indiretos em modelos de mediação (Maroco, 2014). O número de re-amostragens empregado foi de 5000 sujeitos. Testou-se, a presença de um efeito mediador da variável bem-estar psicológico na associação entre as variáveis estilo parental democrático e vinculação amorosa, através dos pressupostos do Teste de Sobel. O Teste de Sobel permite analisar a magnitude da predição da variável independente sobre a dependente, assim como o contributo da variável mediadora na relação inicial. Por outras palavras, analisa o contributo da variável mediadora na relação existente entre as variáveis independente e dependente (Baron \& Kenny, 1986).

\section{Resultados}

Variância dos estilos parentais e da vinculação amorosa de jovens adultos em função do sexo, idade, duração da relação amorosa
Tendo como propósito analisar as diferenças dos estilos parentais e da vinculação amorosa de jovens adultos em função das dimensões sociodemográficas da amostra, efetuaram-se análises de variância uni e multivariada (Teste-t face ao sexo, MANOVA e Análises de discriminatória face à idade). No que concerne aos estilos parentais, os resultados indicaram diferenças significativas face ao sexo e face à idade. As análises univariadas apontam que estas diferenças ocorrem nas escalas apoio $t_{(785)}=-3,87, p=0,001$, IC $95 \%[-0,50$, $-0,16], t_{(785)}=-4,36, p=0,001$, IC $95 \%[-0,43$, $-0,16]$ face ao pai e à mãe, cedência de autonomia e participação democrática $t_{(785)}=-2,95, p=$ 0,003, IC $95 \%[-0,39,-0,08], t_{(785)}=-4,14, p=$ 0,001 , IC $95 \%[-0,42,-0,15]$ face ao pai e à mãe, coerção punição $t_{(337,53)}=3 ; 50, p=0,001$, IC $95 \%$ $[0,06,0,23], t_{(352.79)}=3,20, p=0,002$, IC $95 \%$ $[0,05,0,22]$ face ao pai e à mãe. O sexo feminino evidencia uma percepção mais elevada de apoio, cedência de autonomia e participação democrática, face ao pai e à mãe, comparativamente ao sexo masculino. Relativamente à coerção punição, o sexo masculino evidencia uma percepção mais elevada, face ao pai e à mãe, comparativamente ao sexo feminino (tabela 1).

No que respeita à idade, foram estabelecidos grupos (18 a 20 anos; 21 a 25 anos) para se proceder às análises diferenciais, sendo que os resultados apontam para a presença de diferenças estatisticamente significativas nas escalas cedência de autonomia e participação democrática $F_{(1,785)}=$ $5,42, p=0,02, \eta^{2}=0,993, F_{(1,785)}=4,76, p=0,03$, $\eta^{2}=0,994$, face ao pai e à mãe, indulgência $F_{(1,785)}$ $=4,26, p=0,04, \eta^{2}=0,995$ face ao pai. Os jovens adultos com idades compreendidas entre os 18 e os 20 anos evidenciam uma percepção mais elevada de cedência de autonomia e participação democrática, face ao pai e à mãe, comparativamente com os jovens adultos com idades compreendidas entre os 21 a 25 anos. Relativamente à indulgência, os jovens adultos com idades entre os 18 a 20 anos evidenciam uma percepção mais elevada, face ao 
pai, comparativamente com os jovens com idades compreendidas entre os 21 e 25 anos (tabela 2).

No que concerne à vinculação amorosa, os resultados indicaram diferenças significativas face ao sexo. Contudo, não foram encontradas diferenças estatisticamente significativas desta variável face à idade. Estas diferenças são assinaláveis nas escalas confiança $t_{(785)}=-5,78, p=0,001$, IC $95 \%[-0,46$, - 0,23], evitamento $t_{(785)}=6,12, p=0,001$, IC $95 \%$ $[0,28,0,54]$ e ambivalência $t_{(785)}=3,49, p=0,001$, IC $95 \%[0,14,0,43]$. O sexo feminino evidencia uma percepção mais elevada de confiança compa- rativamente ao sexo masculino. Contudo, o sexo masculino evidencia perceções mais elevadas de evitamento e ambivalência, comparativamente ao sexo feminino (tabela 1). As análises multivariadas sugerem a presença de diferenças estatisticamente significativas da vinculação amorosa face à idade $F_{(4,782)}=3,09, p=0,02, \eta^{2}=0,81$, contudo, não ocorrem diferenças estatisticamente significativas nas escalas confiança $F_{(1,785)}=2,48, p=0,12, \eta^{2}=$ 0,349 , dependência $F_{(1,785)}=0,18, p=0,68, \eta^{2}=$ 0,070 , evitamento $F_{(1,785)}=0,08, p=0,78, \eta^{2}=0,06$ e ambivalência $F_{(1,785)}=0,84, p=0,36, \eta^{2}=0,15$.

Tabela 1

Comparação de médias da vinculação amorosa em função do sexo

\begin{tabular}{|c|c|c|c|c|}
\hline Dimensões & $\begin{array}{c}\text { Masculino } \\
(\mathrm{n}=233) \\
M \pm D P\end{array}$ & $\begin{array}{c}\text { Feminino } \\
(\mathrm{n}=554) \\
M \pm D P\end{array}$ & IC $95 \%$ & $\begin{array}{c}\text { Direção das diferenças } \\
\text { significativas }\end{array}$ \\
\hline \multicolumn{5}{|l|}{ Estilos Parentais Pai } \\
\hline Apoio & $3.19 \pm 1.04$ & $3.52 \pm 1.13$ & {$[-.50,-.16]$} & $1<2$ \\
\hline Cedência de autonomia e participação democrática & $3.25 \pm .97$ & $3.49 \pm 1.04$ & {$[-.39,-.08]$} & $1<2$ \\
\hline Coerção Punição & $1.53 \pm .59$ & $1.38 \pm .43$ & {$[.07, .23]$} & $1>2$ \\
\hline \multicolumn{5}{|l|}{ Estilos Parentais Mãe } \\
\hline Apoio & $3.75 \pm .85$ & $4.05 \pm .89$ & {$[-.43,-.16]$} & $1<2$ \\
\hline Cedência de autonomia e participação democrática & $3.51 \pm .87$ & $3.79 \pm .87$ & {$[-.42,-.15]$} & $1<2$ \\
\hline Coerção Punição & $1.53 \pm .58$ & $1.40 \pm .44$ & {$[.05, .23]$} & $1>2$ \\
\hline \multicolumn{5}{|l|}{ Vinculação amorosa } \\
\hline Confiança & $4.84 \pm .81$ & $5.18 \pm .75$ & {$[-.46,-.23]$} & $1<2$ \\
\hline Evitamento & $2.69 \pm .86$ & $2.28 \pm .85$ & {$[.28, .54]$} & $1>2$ \\
\hline Ambivalência & $2.62 \pm .92$ & $2.33 \pm .97$ & {$[.14, .43]$} & $1>2$ \\
\hline
\end{tabular}

Tabela 2

Comparação de médias dos estilos parentais amorosa em função da idade

\begin{tabular}{|c|c|c|c|}
\hline Dimensões & Idade & $\mathrm{M} \pm \mathrm{DP}$ & Direção das diferenças significativas \\
\hline \multicolumn{4}{|l|}{ Estilos Parentais Pai } \\
\hline Cedência de autonomia e participação democrática & $\begin{array}{l}1-18 \text { a } 20 \\
2-21 \text { a } 25\end{array}$ & $\begin{array}{r}3.48 \pm .99 \\
3.30 \pm 1.06\end{array}$ & $1>2$ \\
\hline Indulgência & $\begin{array}{l}1-18 \text { a } 20 \\
2-21 \text { a } 25\end{array}$ & $\begin{array}{r}1.83 \pm .67 \\
1.73 \pm .67\end{array}$ & $1>2$ \\
\hline \multicolumn{4}{|l|}{ Estilos Parentais Mãe } \\
\hline Cedência de autonomia e participação democrática & $\begin{array}{l}1-18 \text { a } 20 \\
2-21 \text { a } 25\end{array}$ & $\begin{array}{l}3.75 \pm .86 \\
3.61 \pm .91\end{array}$ & $1>2$ \\
\hline
\end{tabular}




\section{Efeito dos estilos parentais na vinculação amorosa: papel mediador do bem-estar psicológico}

Foi realizado um teste inicial do modelo, recorrendo a todas as variáveis da vinculação amorosa, todavia apenas as variáveis dependência e ambivalência se mostraram significativas. Assim, a análise de equações estruturais permitiu observar um efeito inicial positivo do estilo parental democrático pai no desenvolvimento de dependência $(\beta$ $=0,11)$ e negativo no desenvolvimento de ambivalência $(\beta=-0,26)$. De igual modo, foi possível verificar que o estilo parental democrático pai prediz positivamente o bem-estar psicológico $(\beta=$ $0,33)$. Testou-se a presença de um efeito mediador da variável bem-estar psicológico na associação entre as variáveis estilo parental democrático e vinculação amorosa, através dos pressupostos do Teste de Sobel (Tabela 3). No modelo final, após introduzida a variável mediadora do bem-estar psicológico, atestou-se que o efeito inicial direto do estilo parental democrático pai sobre a dependência $(\beta=0,11)$ perde magnitude, embora se mantenha significativo $(\beta=.10)$, pelo que se observa uma mediação parcial negativa $\left(S E{ }^{1}=\right.$ $0,04, p<0,001, \beta=-0,043$, IC $90 \%[-0,07,-0,02]$ ), constando-se uma predição indireta negativa de $45,7 \%\left(\mathrm{R}^{2}=0,457\right)$ através do mediador. Os resultados mostraram ainda que o efeito inicial direto do estilo parental democrático pai sobre a ambivalência $(\beta=-0,26)$ perde magnitude $(\beta=$ -0,19), observando-se também uma mediação parcial negativa $(S E=0,04, p<0,001, \beta=-0,036, \mathrm{IC}$ $90 \%[-0,05,-0,02])$, existe uma predição indireta negativa de $15,8 \%\left(\mathrm{R}^{2}=0,158\right)$ através do mediador. Os passos da análise de mediação podem ser vistos na tabela 3 . No que concerne aos índices de ajustamento, estes encontram-se adequados $x_{i(81)}^{2}=$ $481,32, p=0,001$, $\mathrm{CFI}=0,94$; $\mathrm{RMR}=0,05$; RMSEA $=0,08$ (figura 1$)$.

SE: standard error
Tabela 3

Efeito mediador do bem-estar psicológico na associação entre estilos parentais pai e vinculação amorosa

\begin{tabular}{|c|c|c|c|c|}
\hline Sobel Test Steps & B & SE & $\beta$ & $\mathrm{p}$ \\
\hline $\begin{array}{l}1^{\circ} \text { Passo - Estilo parental de- } \\
\text { mocrático pai Dependência }\end{array}$ & .11 & .04 & .11 & .004 \\
\hline $\begin{array}{l}1^{\circ} \text { Passo - Estilo parental de- } \\
\text { mocrático pai Ambivalência }\end{array}$ & -.27 & .04 & -.26 & $<.001$ \\
\hline $\begin{array}{l}2^{\circ} \text { Passo - Estilo parental de- } \\
\text { mocrático pai } \\
\text { Bem-estar psicológico }\end{array}$ & .18 & .02 & .34 & $<.001$ \\
\hline $\begin{array}{l}3^{\circ} \text { Passo - Bem-estar psicoló- } \\
\text { gico - Dependência }\end{array}$ & -.23 & .08 & -.12 & .003 \\
\hline $\begin{array}{l}3^{\circ} \text { Passo - Bem-estar psicoló- } \\
\text { gico - Ambivalência }\end{array}$ & -.33 & .08 & -.16 & $<.001$ \\
\hline $\begin{array}{l}4^{\circ} \text { Passo - Estilo parental de- } \\
\text { mocrático pai } \\
\text { Bem-estar psicológico - De- } \\
\text { pendência }\end{array}$ & -.14 & .04 & -.043 & $<.001$ \\
\hline $\begin{array}{l}4^{\mathrm{o}} \text { Passo - Estilo parental de- } \\
\text { mocrático pai } \\
\text { Bem-estar psicológico - Am- } \\
\text { bivalência }\end{array}$ & -.19 & .04 & -.036 & $<.001$ \\
\hline
\end{tabular}

No modelo inerente à mãe foi igualmente realizada um teste inicial do modelo, recorrendo a todas as variáveis da vinculação amorosa. Todavia apenas as variáveis confiança, ambivalência e evitamento se mostraram significativas. Relativamente à figura materna, a análise de equações estruturais permitiu observar um efeito inicial positivo do estilo parental democrático mãe no desenvolvimento de confiança $(\beta=0,24)$. Constatou-se também a existência de um efeito inicial negativo do estilo parental democrático mãe no desenvolvimento de ambivalência $(\beta=-0,17)$ e de evitamento $(\beta=-0,12)$. De igual modo, foi possível verificar que o estilo parental democrático mãe prediz positivamente o bem-estar psicológico $(\beta=0,33)$. Testou-se a presença de um efeito mediador da variável bem-estar psicológico na associação entre as variáveis estilo parental democrático e vinculação amorosa, através dos pressupostos do Teste de Sobel (Tabela 4). No modelo final, após introduzida a variável media- 


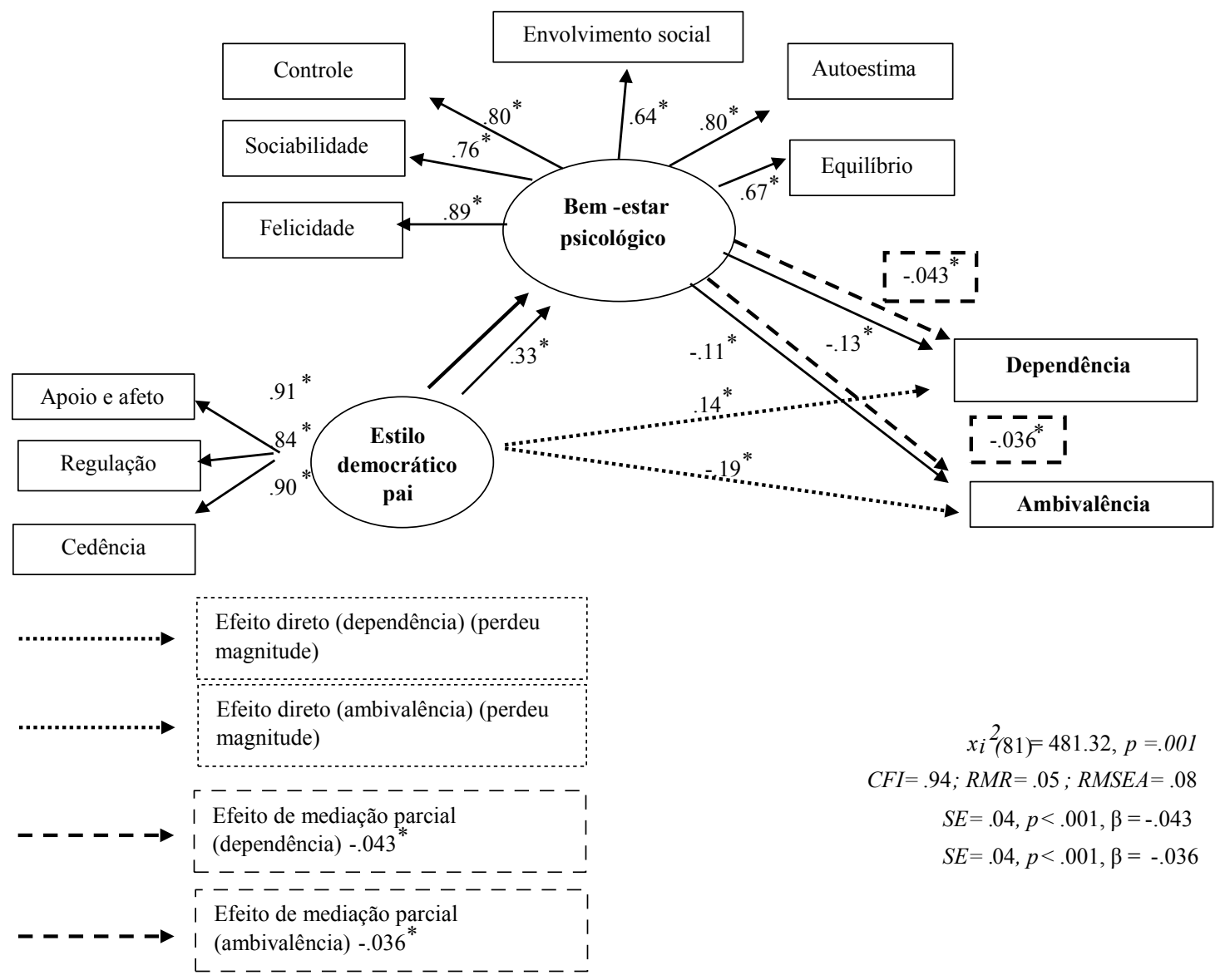

Figura 1. Modelo representativo do efeito mediador do bem-estar psicológo na associação entre o estilo parental democrático pai e vinculção amorosa

dora do bem-estar psicológico, atestou-se que o efeito inicial direto do estilo parental democrático mãe sobre a confiança $(\beta=0,24)$ perde magnitude $(\beta=0,13)$ observando-se uma mediação parcial positiva $(S E=0,06, p=0,003, \beta=0,039$, IC $90 \%$ $[0,015,0,064])$, constata-se uma predição indireta positiva de $22,7 \%\left(\mathrm{R}^{2}=0,227\right)$ através do mediador. Quando a variável mediadora é introduzida na equação final, verificou-se ainda que o efeito inicial direto do estilo parental democrático mãe sobre a ambivalência $(\beta=-0,17)$ perde significância $(\beta=-0,20)$, observando-se uma mediação total negativa do bem-estar psicológico $(S E=0,08$, $p<0,001, \beta=-0,068$, IC $90 \%[-0,095,-0,044])$. No que concerne ao efeito inicial direto do estilo parental democrático mãe sobre o evitamento $(\beta=-0,12)$, verificou-se que o mesmo perde signi- ficância $(\beta=-0,09)$, observando-se uma mediação total negativa do bem-estar psicológico $(S E=0,07$, $p=0,014, \beta=-.031$, IC $90 \%[-0,056,-0,008])$. Os passos da análise de mediação podem ser vistos na tabela 4. Os índices de ajustamento, encontram-se adequados $x_{i(78)}^{2}=439,153, p=0,001, \mathrm{CFI}=0,94$; $\mathrm{RMR}=0,06 ;$ RMSEA $=0,08$ (figura 2).

Tabela 4

Efeito mediador do bem-estar psicológico na associação entre estilos parentais mãe e vinculação amorosa

\begin{tabular}{lcccc}
\hline \multicolumn{1}{c}{ Sobel Test Steps } & B & SE & $\beta$ & $\mathrm{p}$ \\
\hline $\begin{array}{l}1^{\mathrm{o}} \text { Passo - Estilo parental } \\
\text { democrático mãe Confiança }\end{array}$ & .25 & .04 & .24 & $<.001$ \\
$\begin{array}{l}1^{\mathrm{o}} \text { Passo - Estilo parental } \\
\text { democrático mãe Ambi- }\end{array}$ & -.21 & .05 & -.17 & $<.001$
\end{tabular}




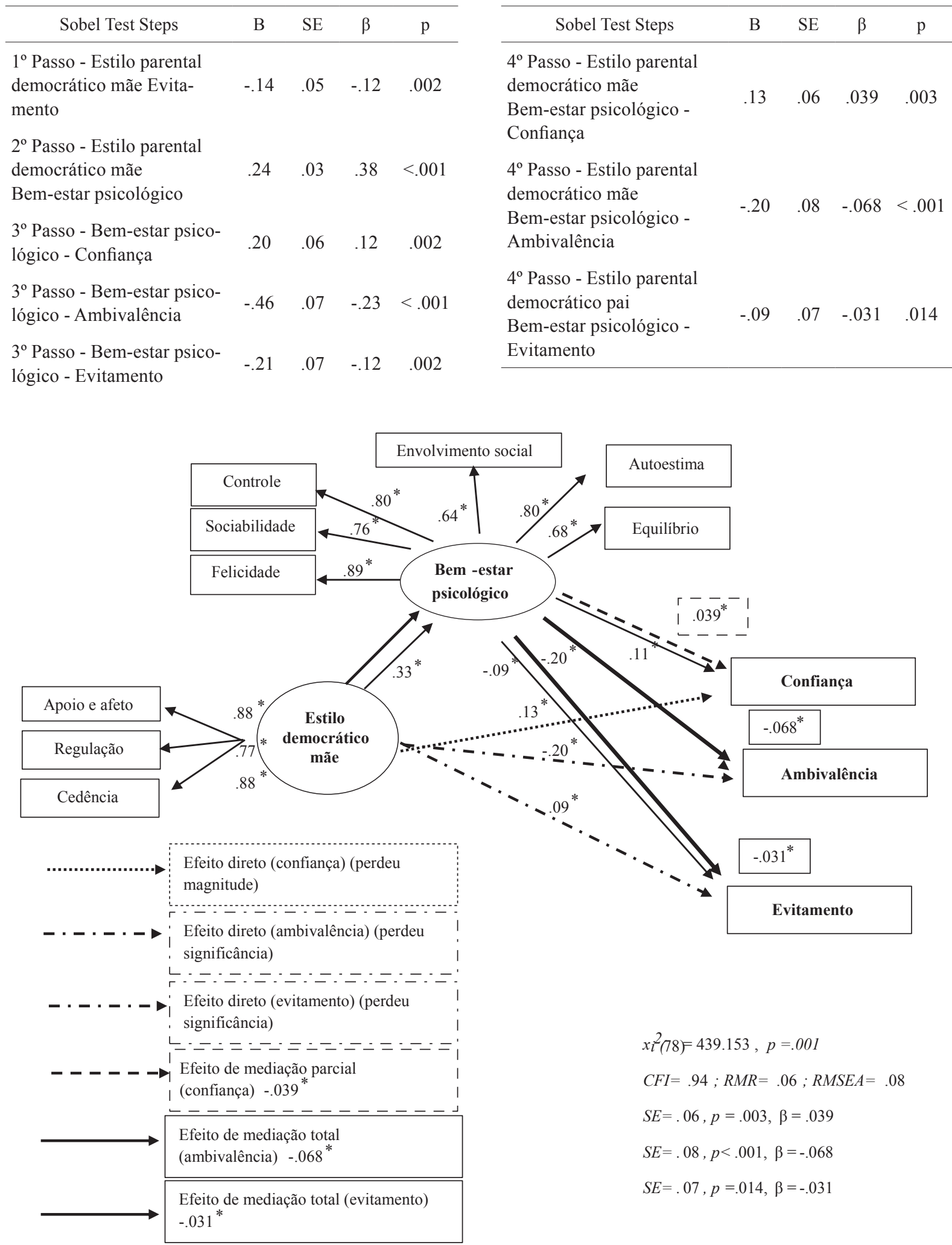

Figura 2. Modelo representativo do efeito mediador do bem-estar psicológo na associação entre o estilo parental democrático mãe e vinculção amorosa 


\section{Discussão}

O presente estudo teve como objetivo testar o papel dos estilos parentais e do bem-estar psicológico na vinculação amorosa. Pretendeu-se ainda analisar o efeito mediador do bem-estar psicológico na associação entre os estilos parentais e a vinculação amorosa. Os resultados do presente estudo reportam a presença de diferenças estatisticamente significativas da vinculação amorosa relativamente ao sexo, tendo-se verificado que os homens evidenciam níveis mais elevados de evitamento e ambivalência do que as mulheres. Por outro lado, as mulheres apresentam níveis mais elevados de confiança comparativamente aos homens. Estes resultados evidenciam as diferenças de gênero na vinculação ao par romântico, verifica-se que as mulheres se mostram mais disponíveis, pretendem uma maior proximidade com o par romântico, o que se traduz na confiança. De acordo com Matos (2002), as mulheres apresentam maior confiança quando envolvidas em uma relação romântica; pelo contrário, os homens revelam-se mais evitantes nas suas relações. No que concerne ao sexo, os resultados apontam para a presença de diferenças estatisticamente significativas nos estilos parentais. Nesta medida, o sexo feminino apresentou uma percepção mais elevada de apoio e cedência de autonomia e participação democrática face ao pai e à mãe, comparativamente com o sexo masculino que tem uma percepção mais elevada de coerção punição face ao pai e à mãe. Pelo exposto, verifica-se um maior investimento parental percepcionado pelo sexo feminino. Estes resultados são consistentes com a literatura onde é sugerido que nos homens comparativamente com as mulheres, é reportada uma maior pontuação para os estilos parentais mais negativos (autoritário e permissivo) (e.g. Brand et al., 2011; Olivari, Wahn, Maridaki-Kassotaki, Antonopoulou \& Confalonieri, 2015). Hutz \& Bardagir (2006) constataram que o sexo masculino se mostra mais suscetível à influência parental, estando desta forma, mais atento aos estilos parentais. No que respeita aos estilos parentais face à idade, os resultados apontam para a presença de diferenças estatisticamente significativas nas escalas cedência de autonomia e participação democrática, face ao pai e à mãe, e na escala indulgência face ao pai. Neste sentido, os jovens adultos com idades compreendidas entre os 18 e os 20 anos apresentam uma maior percepção de cedência de autonomia e participação democrática, face ao pai e à mãe, e uma maior percepção de indulgência face ao pai, comparativamente com os jovens com idades compreendidas entre os 21 e os 25 anos. Face aos resultados da presente amostra, sugere-se que a cedência de autonomia e o suporte emocional por parte das figuras parentais tenda a ser mais reconhecido pelo jovem quando este inicia a sua adolescência (e.g., Meeus, Iedema, Maassen \& Engels, 2005). Desta forma, com o aumento da idade, os jovens desenvolvem a sua autonomia e as figuras parentais tendem a diminuir o controle exercido sobre os jovens, controle esse que tendem a exercer nos mais novos. Por conseguinte, com o aumento da idade, o jovem adulto desenvolve uma menor percepção de cedência de autonomia e participação democrática, assim como de indulgência. Segundo alguns autores, grande parte das figuras parentais tendem a diminuir os comportamentos de controle e supervisão ao longo do período de desenvolvimento do jovem, podendo relacionar-se com níveis de maior autonomia e segurança dos jovens (e.g. Elstad \& Stefansen, 2014; Steinberg $\&$ Morris, 2001).

Relativo às diferenças da vinculação amorosa face à idade, também não foram encontradas diferenças significativas nas subescalas. Seria esperado que os jovens adultos mais velhos evidenciassem uma maior vinculação amorosa, uma vez que se encontram numa fase de maior maturidade emocional que propicia o desenvolvimento de relações amorosas mais estáveis e satisfatórias (e.g. Gleeson \& Fitzgerald, 2014; Matos \& Costa, 2006; Muraru $\&$ Turluc, 2012). Contudo, uma vez que existe uma proximidade etária entre os grupos e uma parti- 
lha de contextos vivenciais, poderá ter ocasionado que estas diferenças não fossem encontradas. Os resultados permitiram ainda verificar que, tal como esperado, um estilo parental democrático prediz positivamente o bem-estar psicológico (e.g. Buist, Dekovic, Meeus \& VanAken, 2002; Veld \& Denollet, 2011). Os resultados apontam que os jovens adultos que vivenciaram um ambiente democrático tendem a ter níveis mais elevados de bem-estar psicológico ao nível da autoestima, controle de si e dos acontecimentos, envolvimento social, equilíbrio, felicidade e sociabilidade. Neste sentido, um ambiente democrático parece ser um fator importante no estabelecimento de um maior bem-estar psicológico na fase adulta. Esta perspectiva parece ser corroborada pelo estudo de Boeckel \& Sarriera (2006), que pretendeu testar o efeito dos estilos parentais, estilos atribucionais e bem-estar psicológico em 323 jovens universitários de ambos os sexos. Os investigadores também concluíram que o estilo parental democrático prediz o bem-estar psicológico.

Por fim, destaca-se nos resultados obtidos, o papel do bem-estar psicológico enquanto variável mediadora na associação entre um estilo parental democrático e a vinculação amorosa. $\mathrm{O}$ efeito mediador do bem-estar psicológico permite verificar que o estilo parental democrático pai prediz negativamente a ambivalência e a dependência e um estilo parental democrático mãe prediz negativamente o evitamento e a ambivalência, e positivamente a confiança. Desta forma, o fato de os jovens adultos serem educados pela figura paterna num estilo parental democrático, parece atuar como fator protetor ao nível do desenvolvimento de dependência e evitamento na relação amorosa. Os jovens adultos com um estilo parental democrático e cujo bem-estar psicológico é positivo, denotam uma diminuição de relações amorosas pautadas por dependência, ambivalência e evitamento, traduzindo uma maior percepção de confiança no par romântico. Ou seja, os jovens adultos que vivenciaram um ambiente pautado por confiança, comunicação clara, respeito e valorização, tendem a desenvolver um maior bem-estar psicológico, o que se manifesta na relação amorosa, neste caso, traduz-se numa maior confiança no par. Por outro lado, a figura materna parece propiciar o desenvolvimento de confiança na relação amorosa do jovem adulto, e atuar como fator protetor ao nível do desenvolvimento de evitamento e ambivalência na relação amorosa. Desta forma, apesar de ambas as figuras parentais adotarem um estilo parental democrático, parece existir uma certa convergência na forma como praticam este estilo parental. Estes resultados são consistentes com a literatura, uma vez que um clima de apoio, regulação e participação democrática na relação com os pais, pode contribuir para níveis mais elevados de bem-estar psicológico no jovem adulto, estando mais predisponente para o desenvolvimento de relações amorosas saudáveis (e.g. Fraley, 2002; Monteiro et al., 2007; Waters \& Cummings, 2000). Também se verifica que os jovens adultos com uma maior percepção de bem-estar psicológico são aqueles que mais confiam no parceiro amoroso. Por outro lado, os jovens adultos com maior evitamento, dependência e ambivalência para com o parceiro amoroso, são aqueles que perceberam menores índices de bem-estar psicológico (e.g. Murray, Holmes \& Griffin, 2000; Veld \& Denollet, 2011). Parece verificar-se que quando o jovem adulto percebe um maior bem-estar psicológico, esta percepção reflete-se na sua vida, nomeadamente, na sua relação amorosa. Por outro lado, também a relação amorosa poderá atuar no sentido de aumentar ou diminuir a percepção de bem-estar psicológico por parte do jovem adulto. Por fim, verifica-se que a atuação de um estilo parental democrático juntamente com um bem-estar psicológico positivo dos jovens adultos, parecem ser fatores fundamentais para o desenvolvimento de uma vinculação amorosa pautada por confiança. 


\section{Implicações práticas, limitações e pistas futuras}

O presente estudo permitiu conhecer os efeitos que a adoção de estilos parentais podem assumir no desenvolvimento de confiança, evitamento, dependência e ambivalência face ao par romântico. Este estudo também permitiu conhecer os efeitos que a vinculação amorosa traduz no bem-estar psicológico dos jovens adultos, assim como enfatizar a adoção de condutas parentais não democráticas no desenvolvimento de um menor bem-estar psicológico. Ressalta-se como principal limitação a coleta de dados mediante instrumentos de autorrelato. Uma vez que este estudo é de caráter transversal, impossibilita o estabelecimento de relações de causa-efeito, contudo, torna-se relevante em estudos futuros desenvolver análises longitudinais de forma a testar o processo de desenvolvimento da vinculação amorosa ao longo das etapas da adolescência até a fase adulta. Seria importante desenvolver programas que permitissem a intervenção e prevenção precoces por forma a minimizar as consequências negativas advindas da vinculação amorosa insegura, assim como desenvolver estratégias de coping que permitissem aos jovens adultos lidar com as adversidades e prevenir o desenvolvimento de um menor bem-estar psicológico. O presente estudo poderia beneficiar da inclusão de outros informantes (como os pais e o par amoroso), assim como outras variáveis, nomeadamente a sintomatologia psicopatológica e outras variáveis psicossociais e desenvolvimentais como as competências sociais e o coping. Seria igualmente relevante contemplar uma faixa etária mais abrangente.

\section{Referências}

Ainsworth, M. (1967). Infancy in Uganda: infant care and the growth of love. Baltimore: Johns Hopkins University Press.
Ainsworth, M. (1991). Attachments and other affectional bonds across the life cycle. In C. M. Parkes, J. Hinde-Stevenson \& P. Marris. (Ed.), Attachment across the life cycle (pp. 32-49). London: Routledge.

Ainsworth, M., Blehar, M., Waters, E., \& Wall, S. (1978). Patterns of attachment: Assessed in the strange situation and at home. Hillsdale: Erlbaum.

Altin, M., \& Terzi, S. (2010). How does attachment styles relate to intimate relationship to aggravate the depressive symptoms? Procedia Social and Behavioral Sciences, 2, 1008-1015. doi:10.1016/j.sbspro.2010.03.142

Baron, R., M., \& Kenny, D., A. (1986). The moderator-mediator variable distinction in social psychological research: Conceptual, strategic and statistical considerations. Journal of Personality and Social Psychology, 51, 1173-1182. doi: http://dx.doi.org/10.1037/0022-3514.51.6.1173

Bartholomew, K., \& Shaver, P. R. (1998). Methods of assessing adult attachment: Do they converge? In J. A. Simpson \& W. S. Rholes (Eds.), Attachment theory and close relationships (pp. 25-45). New York: Guildford Press.

Baumrind, D. (1966). Effects of authoritative parental control on child behavior. Child Development, 37 (4), 887-907.

Baumrind, D. (1968). Authoritarian vs. authoritative parental control. Adolescence, 3 (11), 255-272.

Baumrind, D. (1980). New directions in socialization research. American Psychologist, 9, 293-306.

Baumrind, D. (1991). The influence of parenting style on adolescent competence and substance use. The Journal of Early Adolescence, 11(1), 56-95. Baumrind, D., Larzelere, R., \& Owens, E. (2010). Effects of preschool parents' power assertive patterns and practices on adolescent development. Parenting: Science and Practice, 10, 157-201. doi:http://dx.doi. org/10.1080/15295190903290790

Boeckel, M. G., \& Sarriera, J.C. (2006). Estilos parentais, estilos atribucionais e bem-estar 
psicológico em jovens universitários. Revista Brasileira Crescimento Desenvolvimento Humano, 16(3), 53-65.

Bowlby, J. (1969). Attachment and loss. Vol. 1: Attachment. New York: Basic Books.

Bowlby, J. (1973). Attachment and loss: Vol. 2. Separation. New York: Basic Books.

Bowlby, J. (1988). A secure base: parent-child attachment and healthy human. New York: Basic Books.

Brand, S., Gerber, M., Beck, J., Kalak, N., Hatzinger, M., Pühse, U., \& Holsboer-Trachsler, E. (2011). Perceived parenting styles differ between genders but not between elite athletes and controls. Adolescent Health. Medicine and Therapeutics, 2, 9-14. doi:10.2147/AHMT.S16992

Buhl, H. M. (2008). Significance of individuation in adult child-parent relationships. Journal of Family Issues, 29, 262-281. doi:https://doi. org/10.1177/0192513X07304272

Buist, K., Dekovic, M., Meeus, W., \& Van Aken, M. (2002). Developmental patterns in adolescent attachment to mother, father and sibling. Journal of Youth and Adolescence, 1(3), 167-176. doi:10.1023/A:1015074701280

Cecconello, A. M., Antoni, C., \& Koller, S. H. (2003). Práticas educativas, estilos parentais e abuso físico no contexto familiar. Psicologia em Estudo, 8, 45-54.

Collins, W., Welsh, D., \& Furman, W. (2009). Adolescent romantic relationships. The Annual Review of Psychology, 60, 631-652.

Cordeiro, R. (2006). A aparência física e amizade íntima na adolescência: Estudo num contexto pré-universitário. Análise Psicológica, 4(24), 509-517.

Dinero, R., Conger, R., Shaver, P., Widaman, K., \& Larsen-Rife, D. (2008). Influence of family of origin and adult romantic partners on romantic attachment security. Journal of Family Psychology, 22(3), 622-632. doi: 10.1037/a0012506

Dornbusch, S. M., Ritter, P. L., Leiderman, P. H., Roberts, D. E., \& Fraleigh, M. J. (1987). The relation of parenting style to adolescence school performance. Child Development, 58, 12441257.

Elstad, J., \& Stefansen, K. (2014). Social variations in perceived parenting styles among norwegian adolescents. Child Indicators Research, 7(3), 649-670. doi:10.1007/s12187-014-9239-5

Fraley, R. (2002). Attachment stability from infancy to adulthood: Meta-analysis and dynamic modeling of developmental mechanisms. Personality and Social Psychology Review, 6(2), 123-151.

Gleeson, G., \& Fitzgerald, A. (2014). Exploring the association between adult attachment styles in romantic relationships, perceptions of parents from childhood and relationship satisfaction. Health, 6, 1643-1661. doi: 10.4236/ health.2014.613196.

Hazan, C., \& Shaver, P. R. (1990). Love and work: an attachment-based theoretical perspective. Journal of Personality and Social Psychology, 52, 511-524.

Hutz, C., S., \& Bardagir, M., P. (2006). Indecisão profissional, ansiedade e depressão na adolescência: a influência dos estilos parentais. Psico-USF, 11(1), 65-73.

Koepke, S., \& Denissen, J. (2012). Dynamics of identy development and separation-individuation in parentchild relationships during adolescence and emerging adultood - A conceptual integration. Developmental Review, 32, 67-88. doi: 10.1016/j.dr.2012.01.001

Komidar, L., Zupančič, M., Sočan, G., \& Puklek Levpušček, M. (2014). Development and construct validation of the Individuation Test for Emerging Adults (IT-EA). Journal of Personality Assessment, 96, 503-514. doi: 10.1080/00223891.2013.850703

Lamborn, S. D., Mounts, N., Steinberg, L., \& Dornbusch, S.M. (1991). Patterns of competence and adjustment of adolescents from authoritative, authoritarian, indulgent, and neglectful families. Child Development, 62, 1049-65. 
Maccoby, E., \& Martin, J. (1983). Socialization in the context of the family: Parent-child interaction. Em E. M. Hetherington (Org.), P. H. Mussen (Org. Série), Handbook of child psychology: Vol. 4. Socialization, personality, and social development ( $4^{\mathrm{a}}$ ed.) (pp. 1-101). New York: Wiley.

Maroco, J. (2007). Análise estatística com utilização do SPSS. Lisboa: Sílabo.

Maroco, J. (2014). Análise de Equações Estruturais: Fundamentos teóricos, software\& aplicações ( $2^{\mathrm{a}}$ ed.). Pêro Pinheiro: RepotNunber.

Massé, R., Poulin, C., Dassa, C., Lambert, J., Bélair, S., \& Battaglini, A. (1998). Élaboration et validation d'unou til de mesure du bien-être psychologique: L'É.M.M.B.E.P. Revue Canadienne de Santé Publique, 89(5), 352-357.

Matos, M. (2002). (Des)continuidades na vinculação aos pais e ao par amoroso em adolescentes (Tese de Doutoramento, Faculdade de Psicologia, Instituto de Consulta Psicológica, Formação e Desenvolvimento, Universidade do Porto, Porto).

Matos, P. M. (2006). Relações românticas em adolescentes. Psychologica, 41, 9-24.

Matos, P. M. \& Costa, M. E. (2006). Vinculação aos pais e ao par romântico em adolescentes. Psicologia, 20(1), 97-126.

Matos, P. M., Barbosa, S., \& Costa, M.E. (2001). Avaliação da vinculação amorosa em adolescentes e jovens adultos: Construção de um instrumento e estudos de validação. Revista Oficial de la Asociación Iberoamericana de Diagnóstico y Evaluación Psicológica, 11, 93-109.

Meeus, W., Iedema, J., Maassen, G., \& Engels, R. (2005). Separation individuation revisited: On the interplay of parent adolescent relations, identity and emotional adjustment in adolescence. Journal of Adolescence, 28, 89-106. doi: 10.1016/j.adolescence.2004.07.003

Monteiro, S., Tavares, J., \& Pereira, A. (2007). Relação entre vinculação, sintomatologia psicopatológica e bem-estar em estudantes do primeiro ano do ensino superior. Psicologia, Saúde \& Doenças, 8 (1), 83-93.

Mota, C. P., \& Rocha, M. (2012). Adolescência e jovem adultícia: Crescimento pessoal, separação-individuação e o jogo das relações. Psicologia: Teoria e Pesquisa, 28, 357-366. doi: http://dx. doi.org/10.1590/S0102-37722012000300011

Muraru, A. A., \& Turluic, M. N. (2012). Family-of-origin, romantic attachment, and marital adjustment: a path analysis model. Procedia - Social and Behavioral Sciences, 33, 90-94. doi: 10.1016/j.sbspro.2012.01.089

Murray, S. L., Holmes, J. G., \& Griffin, D. W. (2000). Self-esteem and the quest for felt security: how perceived regard regulates Attachment process. Journal of Personality and Social Psychology, 78, 478-498.

Nunes, F., \& Mota, C. P. (in press). Parenting styles and dimensions questionnaire - adaptação da versão portuguesa de heterorrelato [Parenting styles and dimensions questionnaire - Adaptation of Portuguese version]. Revista Colombiana de Psicología, 27(1), XXXX.

Olivari, M., Wahn, E., Maridaki-Kassotaki, K., Antonopoulou, K., \& Confalonieri, E. (2015). Adolescent Perceptions of Parenting Styles in Sweden, Italy and Greece: An Exploratory Study. Europe's Journal of Psychology, 11(2), 244-258. doi:10.5964/ejop.v11i2.887

Pallant, J. (2005). SPSS survival manual: a step by step guide to data analysis using SPSS ( $2^{\mathrm{a} e d}$.). Buckingham: Open University Press.

Pinto, M. (2009). Intimidade em adolescentes de diferentes grupos étnicos. Lisboa: Alto-Comissariado para a Imigração e Diálogo Intercultural.

Rice, K. G., \& Delwo, J. P. (2002). Perfectionism and self-development: implications for college adjustment. Journal of Counseling \& Development, 80 (2), 188-196. doi: 10.1002/j.15566678.2002.tb00182.x

Saraiva, L. M., \& Matos, P. M. (2012). Separation-individuation of Portuguese emerging adults in relation to parents and to the romantic part- 
ner. Journal of Youth Studies, 15, 499-517. doi: 10.1080/13676261.2012.663889

Sarriera, J. C. (1993). Aspectos psicosociales del desempleo juvenil: un análisis desde el fracaso escolar para la intervención preventiva. Madrid: Facultad de Psicología. Universidad Autonomía de Madrid.

Shaver, P. R., \& Hazan, C. (1988). A biased overview of the study of love. Journal of Social and Personal Relationships, 5, 473-510.

Steinberg, L., \& Morris, A. S. (2001). Adolescent development. Annual Review of Psychology, 52, 83-110. doi: 10.1146/annurev.psych.52.1.83

\section{Recebido: Abril 19, 2017}

Aprovado: Agosto 12, 2017
Veld, E. M., \& Denollet, J. J. (2011). Attachment style and self-esteem: The mediating role of type personality. Personality and Individual Differences, 50, 1099 1103. doi: 10.1016/j. paid.2011.01.034

Waters, E., \& Cummings, E. M. (2000). A secure base from which to explore close relationships. Child Development, 71, 164-172. doi: 10.1111/14678624.00130

Williams, N. L., \& Riskind, J. H. (2004). Adult romantic attachment and cognitive vulnerabilities to anxiety and depression: examining the interpersonal basis of vulnerability models. Journal of Cognitive Psychotherapy, 18 (1), 7-24. 
